\title{
Outdoor Augmented Reality Spatial Information Representation
}

\author{
Fu Ren ${ }^{1}$, Xueling $W u^{2, *}$ \\ ${ }^{1}$ School of Resource and Environmental Sciences, Wuhan University, Wuhan 430079, China \\ ${ }^{2}$ Institute of Geophysics and Geomatics, China University of Geosciences, Wuhan 430074, China
}

Received: 26 Oct. 2012, Revised: 3 Feb. 2013, Accepted: 5 Feb. 2013

Published online: 1 Jun. 2013

\begin{abstract}
From the perspective of Location Based Services, this paper explores basic characteristics, architectural design and application mode of outdoor Augmented Reality (AR) spatial information representation. Then system structure is proposed and key technologies are analysed. Finally, two cases are designed to verify the efficiency and accuracy of methods. One puts forward and practices a hybrid hardware registration method. In order to avoid complex and tedious process of position tracking and camera calibration, the other introduces algorithm of computer vision to set up global affine coordinate system for computing projection coordinates of virtual objects into real scene images, and an approach to get the relative depth of pixels is put forward. The results have proved outdoor AR spatial information representation to be an effective optimal method in helping people obtain a vivid and deep-level spatial cognition.
\end{abstract}

Keywords: outdoor Augmented Reality (AR), spatial information representation, virtual-real registration, spatial affine transformations

\section{Introduction}

Augmented Reality (AR) is the registration of projected computer-generated images over a user's view of the physical world, which has three properties: combines real and virtual objects in a real environment, runs interactively in real time, registers (aligns) real and virtual objects with each other [1]. Despite its potential, AR has not received nearly the amount of attention paid to its sibling, Virtual Reality (VR). Most research has focused on indoor environment. Some work along this direction has been done to reduce calibration requirements and need to know geometry of all observed objects at the start [2]. Making AR systems work outdoor is a natural step in the development of AR toward the ultimate goal of AR displays that can operate anywhere, in any environment [3].

Using AR to visualize geospatial information in outdoor environments has just started. Most applications in this area are focusing on systems which deliver information to tourists [4]. Portalés et al. introduced a low-cost outdoor mobile AR application to integrate buildings of different urban spaces [5]. Hedley et al. describe explorations in the use of AR for geographic visualization [6]. Du et al. discuss key technologies of integrate outdoor AR with mobile Geographic information system (GIS) [7].

The above researches have made some fruitful results in introduction outdoor AR into GIS. However, there are still some problems to solve in outdoor AR spatial information representation. This paper expatiates basic characteristics, architectural design and application mode of outdoor AR spatial information representation. System structure is proposed. Virtual-real registration is the key of outdoor AR spatial information representation, and two ideas are used to solve it. Examples are designed to verify the efficiency of methods.

\section{Outdoor AR spatial information representation}

\subsection{Basic theory}

Spatial cognition is one of goals of GIService. People acquire spatial knowledge in many different ways. How

\footnotetext{
*Corresponding author e-mail: snowforesting@163.com
} 
to make the way of spatial information access and browse meet people's daily behavior and close to people's habits of mind has become the focus of attention. Spatial information visualization consists of Geo-visualization, Virtual Geographic Environment and outdoor AR. From GIS visualization to outdoor AR, it requires not only overlaying virtual geographic object into real scene, but also positioning accurately virtual objects in the scene with a sense of realism and location. User-centric spatial information services and interaction pay more attention to supplement reality, rather than completely replace it. It would appear to user the virtual-real coexisting environment.

\subsection{Main characteristics}

Relation between outdoor $\mathrm{AR}$ and spatial information representation embodies Location Based Service (LBS). Emergence of mobile GIS provides powerful technical channel to outdoor AR spatial information representation, which has the following characteristics: Mobility is foremost in outdoor AR, which is distinct from indoor AR. Real-time requires system with low latency. Natural interaction between users and virtual-real coexisting environment is necessary. 3D modeling must be effective. With robustness, the system could work in outdoor environment. Wireless network allows users to obtain geospatial information real-time in a wide range.

\subsection{Architectural design}

Outdoor AR spatial information representation provides users with LBS by overlaying virtual objects into the real environment. The basic architectural design is shown in Figure 1. It still exist three questions to solve. Virtual-real registration emphasis location accuracy of virtual-real spatial information overlay, which depends on precision of features points of virtual geographical object model and their attributes projected to image of the real scene. Correct registration of a virtual image with the real scene requires the same frame of reference. Virtual-real combination shows requirements in display quality and effectiveness, which display virtual objects and real scene in a specific display with illumination consistency, occlusion and seamless stitching results. Naturally interact with virtual objects is also one of goals of outdoor AR.

\subsection{Application mode}

Outdoors AR makes new application areas possible and provides a natural interface for spatial information visualization. Outdoor AR help user detect automatically objects who need information about their current

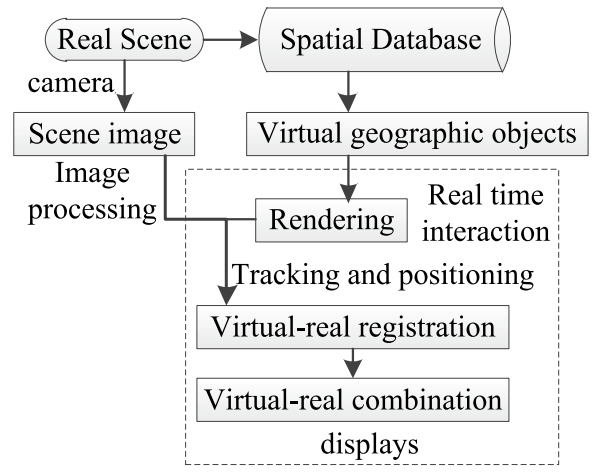

Fig. 1 Architectural design

environment to navigate and identify features of interest. This information can be text labels, arrows or even 3D objects. All outdoor AR applications can be seen as LBS. Software environment of outdoor AR is composed of four application levels shown in Figure 2: basic platform layer is the lowest layer of system. Platform layer provides the necessary devices and functions. The above two layers are independent of application software layer and content layer. Application layer runs on the platform layer and is independent of content layer.

\begin{tabular}{|l|l|}
\hline $\begin{array}{l}\text { Content layer } \\
\text { Software layer }\end{array}$ & Spatial information DB \\
\cline { 2 - 2 } & Application of outdoor AR \\
\cline { 2 - 2 } Platform layer & $\begin{array}{l}\text { HMD, Virtual-real registration, } \\
\text { Merger class library }\end{array}$ \\
$\begin{array}{l}\text { Basic } \\
\text { layer }\end{array}$ & $\begin{array}{l}\text { Computer, OS , class library, } \\
\text { hardware and other related devices }\end{array}$ \\
\hline
\end{tabular}

Fig. 2 Four application levels

\section{Methods}

\subsection{Virtual-real registration based on hybrid hardware tracking and location}

Considering natural attribute of virtual-real registration, direct method is to define some coordinate systems and calculate conversion relationship between them. Only make sure five coordinates systems and four coordinate transformation relationships shown in Figure 3, enhanced images can be generated by registration virtual objects properly into real scene.

Coordinate is the only link to integrate $3 \mathrm{D}$ virtual objects with 2D images and graphics. Coordinate systems 


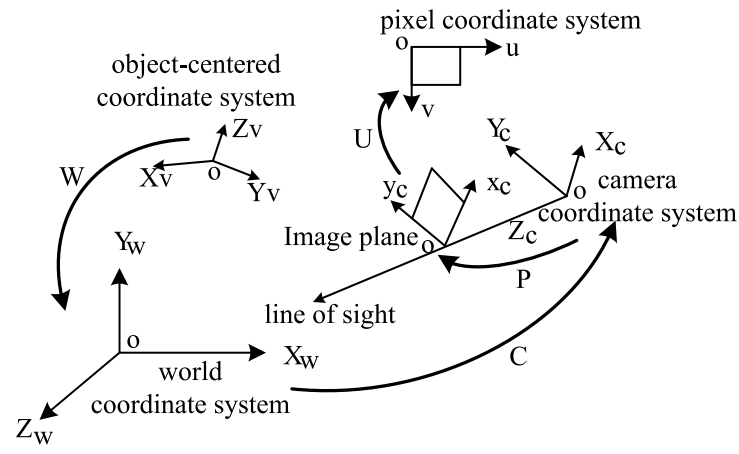

Fig. 3 Five spatial coordinate systems and four conversion relationships

are as followings: $\mathrm{O}-\mathrm{X}_{v} \mathrm{Y}_{v} \mathrm{Z}_{v}$ is virtual object-centered coordinate system, which is used to define three-dimensional coordinates of virtual objects. $\mathrm{O}-\mathrm{XwYwZw}$ is world coordinate system to describe absolute coordinates of geographic objects in the real scene. $\mathrm{O}-\mathrm{XcY} \mathrm{CZc}$ is camera coordinate system to indicate $3 \mathrm{D}$ space coordinate system observed from the user point of view. $\mathrm{O}-\mathrm{x}_{c} \mathrm{y}_{c}$ is a $2 \mathrm{D}$ image plane coordinate system. $\mathrm{O}-\mathrm{uv}$ is pixel coordinate system to describe pixel coordinates in the image array. Transformation relationships are object-to-world $\mathrm{W}$, world-to-camera $\mathrm{C}$, camera-to-image plane $\mathrm{P}$, image-to-pixel $\mathrm{U}$. Transformation from object coordinate system $\left(\mathrm{X}_{v} \mathrm{Y}_{v} \mathrm{Z}_{v}\right.$ 1) to pixel coordinate system $(\mathrm{u} \vee 1)$ is shown as [ $\mathrm{u} \mathrm{v} 1]$ $\mathrm{T}=\mathrm{U} \mathrm{P} \mathrm{C} \mathrm{W}\left[\mathrm{X}_{v} \mathrm{Y}_{v} \mathrm{Z}_{v} 1\right] \mathrm{T}$. Then equation (1) can be deduced, with focal length $f$, principal point coordinates ( $\mathrm{u} 0 \mathrm{v} 0$ ), pixel width $\mathrm{dx}$, height $\mathrm{d}_{y}$, and rotation matrix $\mathrm{R}$ and translation matrix $\mathrm{T}$.

$$
Z_{c}\left[\begin{array}{l}
u \\
v \\
1
\end{array}\right]=\left[\begin{array}{cccc}
f / d_{x} & 0 & u_{0} & 0 \\
0 & -f / d_{y} & v_{0} & 0 \\
0 & 0 & 1 & 0
\end{array}\right]\left[\begin{array}{cccc}
r_{11} & r_{12} & r_{13} & t_{1} \\
r_{21} & r_{22} & r_{23} & t_{2} \\
r_{31} & r_{32} & r_{33} & t_{3} \\
0 & 0 & 0 & 1
\end{array}\right]\left[\begin{array}{c}
X w \\
Y w \\
Z w \\
1
\end{array}\right]
$$

\subsection{Virtual-real registration using affine transformations}

In order to avoid complex and tedious process of position tracking and camera calibration, based on observation from Koenderink et al. [8] and Ullman et al. [9] which is given a set of four or more non-coplanar 3D points, the projection of all points in the set can be computed as a linear combination of the projection of just four of the points. Inspired by this view, a global non-Euclidean affine coordinate system is set up by combination virtual object coordinate system, camera coordinate system and world coordinate system. All points in 3D space can be represented by the global affine coordinates based on affine transformation which is defined using location of feature points in image plane. The affine transformation is used to project attribute and spatial information of virtual geographical objects into the real scene.

In global affine coordinate system, each point of virtual object in augmented scene has fixed affine coordinate. $\mathrm{P}_{i}(\mathrm{i}=0 \sim 3)$ are corresponding $2 \mathrm{D}$ projection plane coordinates of four non-coplanar points. Affine coordinates represent the point with respect to the affine basis point. $\mathrm{P}_{x}$ is $2 \mathrm{D}$ plane coordinate of any point in global affine coordinate. $\left(\mathrm{X}_{f} \mathrm{Y}_{f} \mathrm{Z}_{f} 1\right) \mathrm{T}$ indicates global affine coordinate of $\mathrm{P}_{x}$.

Affine reprojection property: projection of any point (up vp 1) $\mathrm{T}$ is represented as affine coordinates $\left(\mathrm{X}_{f} \mathrm{Y}_{f} \mathrm{Z}_{f}\right.$ 1) $\mathrm{T}$ with homogeneous coordinates shown in equation (2). Projection computation of one point in virtual object has been reduced to knowing projections location of affine basis points in a new image and homogeneous affine coordinates of virtual point.

$$
\left[\begin{array}{lll}
u_{p} & v_{p} & 1
\end{array}\right]^{T}=\left[\begin{array}{cccc}
u_{1}-u_{0} & u_{2}-u_{0} & u_{3}-u_{0} & u_{0} \\
v_{1}-v_{0} & v_{2}-v_{0} & v_{3}-v_{0} & v_{0} \\
0 & 0 & 0 & 1
\end{array}\right]\left[\begin{array}{llll}
X_{f} & Y_{f} & Z_{f} & 1
\end{array}\right]^{T}
$$

Affine reconstruction property: Given two views I1 and 12 of the real scene in which the projections of the affine basis points $\mathrm{Pi}(\mathrm{i}=0 \sim 3)$ are known, then affine coordinates $\left(\mathrm{X}_{f} \mathrm{Y}_{f} \mathrm{Z}_{f} 1\right) \mathrm{T}$ of any point $\mathrm{P}$ can be obtained by equation (3). Where $\left(\mathrm{u}_{p}^{j} \mathrm{v}_{p}^{j}\right) \mathrm{T}(\mathrm{j}=1 \sim 2)$ and $\left(\mathrm{u}_{i}^{j} \mathrm{v}_{i}^{j}\right) \mathrm{T}$ $(\mathrm{j}=1 \sim 2, \mathrm{i}=0 \sim 3)$ are projections of point $\mathrm{P}$ and affine basis point, respectively, in image I1 and I2.

$$
\left[\begin{array}{c}
u_{p}^{1} \\
v_{p}^{1} \\
u_{p}^{2} \\
v_{p}^{2}
\end{array}\right]=\left[\begin{array}{cccc}
u_{1}^{1}-u_{0}^{1} & u_{2}^{1}-u_{0}^{1} & u_{3}^{1}-u_{0}^{1} & u_{0}^{1} \\
v_{1}^{1}-v_{0}^{1} & v_{2}^{1}-v_{0}^{1} & v_{3}^{1}-v_{0}^{1} & v_{0}^{1} \\
u_{1}^{2}-u_{0}^{2} & u_{2}^{2}-u_{0}^{2} & u_{3}^{2}-u_{0}^{2} & u_{0}^{2} \\
v_{1}^{2}-v_{0}^{2} & v_{2}^{2}-v_{0}^{2} & v_{3}^{2}-v_{0}^{2} & v_{0}^{2}
\end{array}\right]\left[\begin{array}{c}
X_{f} \\
Y_{f} \\
Z_{f} \\
1
\end{array}\right]
$$

The familiar notions of "image plane" and "viewing direction" are extended to affine representation, which is committed to calculation depth of projection points. Viewing direction D of camera is expressed through cross product in equation (4). Depth information d of projection points needed for hidden surface removal is expressed as $\mathrm{d}=\mathrm{D} \mathrm{T}\left[\mathrm{X}_{f} \mathrm{Y}_{f} \mathrm{Z}_{f} 1\right] \mathrm{T}$.

$$
\begin{aligned}
D & =\left[\begin{array}{l}
u_{1}-u_{0} \\
u_{2}-u_{0} \\
u_{3}-u_{0}
\end{array}\right] \times\left[\begin{array}{l}
v_{1}-v_{0} \\
v_{2}-v_{0} \\
v_{3}-v_{0}
\end{array}\right] \\
& =\left[\begin{array}{l}
\left(u_{2}-u_{0}\right)\left(v_{3}-v_{0}\right)-\left(u_{3}-u_{0}\right)\left(v_{2}-v_{0}\right) \\
\left(u_{3}-u_{0}\right)\left(v_{1}-v_{0}\right)-\left(u_{1}-u_{0}\right)\left(v_{3}-v_{0}\right) \\
\left(u_{1}-u_{0}\right)\left(v_{2}-v_{0}\right)-\left(u_{2}-u_{0}\right)\left(v_{1}-v_{0}\right)
\end{array}\right]
\end{aligned}
$$




\section{Materials and Experiments}

\subsection{Outdoor AR using hybrid hardware tracking and location}

Digital map of Wuhan University is used as geospatial data, which include six thematic layers such as roads, student dormitories, playgrounds, school buildings, staff quarters, and other ancillary facilities. Portable computers are used as display. 2D projection images are captured by CCD camera. User is located by navigation GPS and 3D electronic compass. Software structure is integrated on the basis of Visual Studio.net 2005 platform. Geospatial data are managed using MapObject and simple virtual geographic object models are drawn using OpenGL functions.

Workflow of outdoor AR using hybrid hardware tracking and location is shown in Figure 4. Client transmit position and pose of user's head to server real time, then the server implements map navigation and identifies geographic objects in the field of view. Furthermore, in term of attribute and spatial information of geographic object to modeling by calling OpenGL functions. Finally, it overlay 2D projection image to finish virtual-real combination.

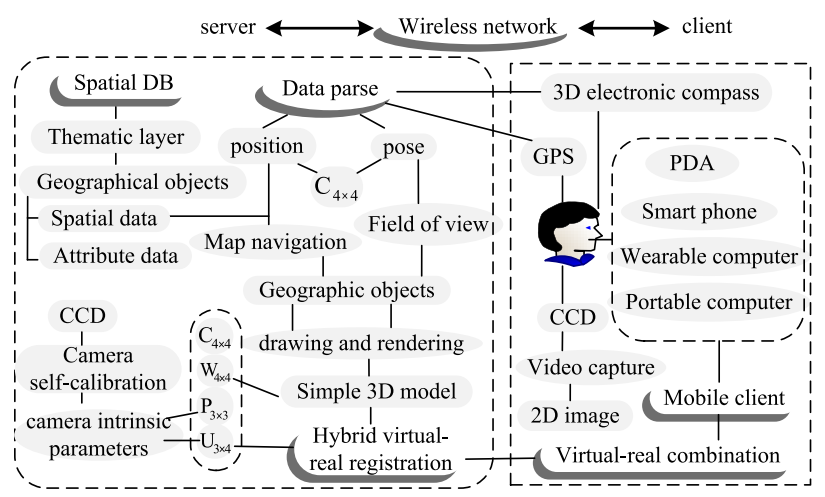

Fig. 4 Workflow of outdoor AR using hybrid hardware tracking and location

\subsection{Outdoor AR using affine representations}

Two images of hydropower branch office in Wuhan University are captured and nineteen same name feature points are extracted, A B C D to set up global affine coordinate system, P E F F1 F2 F3 to register virtual objects and attribute information into real scene, F2.....F12 to evaluate pixel errors. Scene images I and II include A B C D respectively, with image resolution $3648 \times 2736$, width $30.89 \mathrm{~cm}$ and height $23.16 \mathrm{~cm}$. Global affine coordinates of P E F F1 F2 F3 are transformed into image plane coordinates in Figure 5 to augment feature points $\mathrm{P} F$ by attribute information and $\mathrm{E}$ F1 F2 F3 by spatial information.

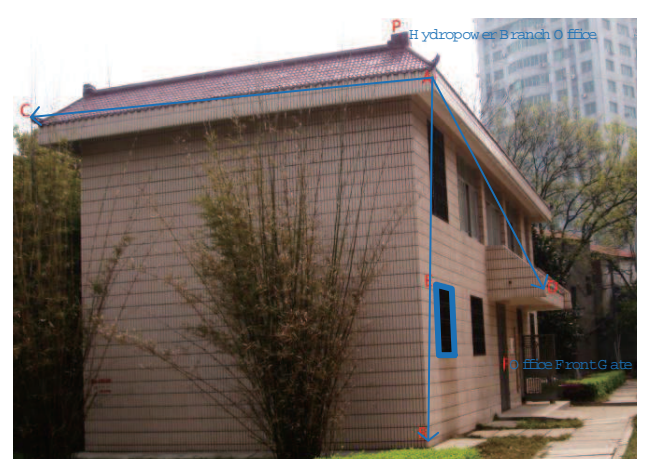

Fig. 5 Virtual-real spatial information visualization registration

Loading more feature points to verify algorithm, pixel coordinates and error of feature points in $\mathrm{U}$ and $\mathrm{V}$ direction can be obtained, shown in Table 1.

Table 1 Pixel error of feature points

\begin{tabular}{lcc}
\hline Points NO. & $\Delta \mathrm{U}$ (pixel) & $\Delta \mathrm{V}($ pixel $)$ \\
\hline E & 0.75 & 1 \\
F & 15.8 & 4.2 \\
P & -5.8 & 2.8 \\
F1 & 10.3 & 1.5 \\
F2 & 4.3 & 4.8 \\
F3 & 8.6 & -2.5 \\
F4 & 0.0 & -6.0 \\
F5 & 5.2 & -6.0 \\
F6 & 9.0 & -8.8 \\
F7 & -0.4 & -0.3 \\
F8 & -1.2 & -0.9 \\
F9 & -2.8 & -1.7 \\
F10 & -1.1 & -1.8 \\
F11 & -1.8 & -2.6 \\
F12 & -5.5 & -2.8 \\
\hline
\end{tabular}

Combination the relative distance with pixel error in $\mathrm{U}$ and $\mathrm{V}$ direction, relation between relative depth and pixel error is shown in Figure 6: distance between camera and virtual object is limited to 200 meters. Registration error is limited to 10 pixels. Pixel width and height are $0.0085 \mathrm{~cm}$, which meet basic demands of outdoor AR. With improvement of projection images resolution, pixel error is also increased. With increasing of distance, registration error is also increased for accuracy decline of feature point. Close observation of geographical object is a prerequisite of using affine spatial transformation to achieve virtual-real registration. 


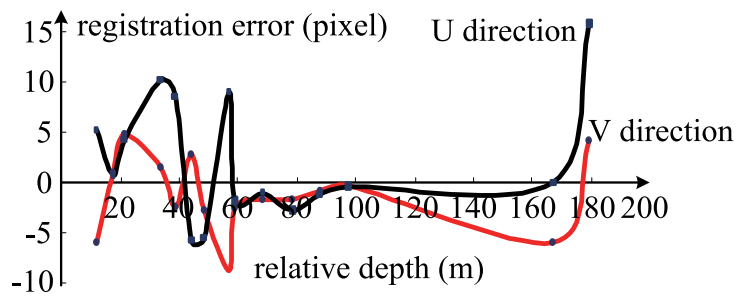

Fig. 6 Relation between relative depth and pixel error

\section{Conclusions}

Traditional spatial information representation cannot satisfy user's demands in visual fidelity and naturalness of Human-computer interaction (HCI). Outdoor AR provides an innovate method to solve these questions. This paper explores architectural design and application mode of outdoor AR spatial information representation, and system structure is analyzed. In addition, virtual-real registration is crucial to outdoor AR spatial information representation and two ideas are used to solve it. Cases are designed to verify accuracy of the methods. Outdoor AR spatial information representation is the result of spatial information representation, and it is also a process. For what the practical effect it is to achieve, it remains to be studied. However, it is not a purely technical system, but a user-centric integrated system.

\section{Acknowledgements}

The research is supported by NSFC (41271455/D0108), Fundamental Research Funds for the Central Universities, China University of Geosciences (Wuhan) (CUGL120207), and Open Research Fund of Key Laboratory of Disaster Reduction and Emergency Response Engineering of the Ministry of Civil Affairs (LDRERE20120207).

\section{References}

[1] R. T. Azuma, Y. Baillot, R. Behringer, S. Feiner, S. Julier, and B. MacIntyre. Recent advances in augmented reality, IEEE Computer Graphics and Applications, (2001), 21(6) , 34-47

[2] K. N. Kutukalosand, J. R. Vallino. Calibration-Free Augmented Reality, IEEE Transactions on Visualization and Computer Graphics, (1998), 4, 1-20

[3] R. T. Azuma. The challenge of making augmented reality work outdoors, In Mixed Reality: Merging Real and Virtual Worlds, (1999), 379-390

[4] D. Holwegand, U. Kretschmer. Augmented reality visualization of geospatial data, In Frontiers of Geographic Information Technology, Eds. S. Rana and J. Sharma, (2006), 229-240
[5] C. Portalés, J. L. Lerma, S. Navarro. Augmented reality and photogrammetry: A synergy to visualize physical and virtual city environments. ISPRS Journal of Photogrammetry and Remote Sensing, (2010), 65, 134-142

[6] N. R. Hedley, M. Billinghurst, L. Postner, R. May, and H. Kato. Explorations in the use of augmented reality for geographic visualization, Teleoperators and Virtual Environments, (2002), 11(2), 119-133

[7] Q. Du and T. Liu. Design and Implementation of a Prototype Outdoor Augmented Reality GIS, Geomatics and Information Science of Wuhan University, (2007), 32(11), 1046-1049

[8] J. J. Koenderink, A. J. Van Doorn. Affine structure from motion, Journal of the Optical Society of America, (1995), 8(2), 377-385

[9] S. Ullman, R. Basri. Recognition by Linear Combinations of Models, IEEE transactions on pattern analysis and machine intelligence, (1991), 13(10), 992-1006

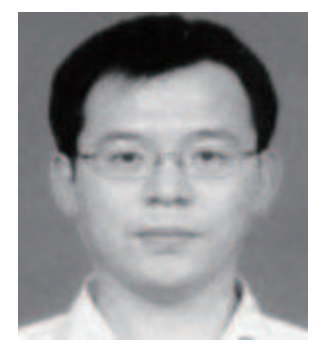

Fu Ren is an associate professor at department of Geographic Information Science in School of Resource and Environmental Sciences, Wuhan University, China. He holds an MA and $\mathrm{PhD}$ in Cartography and Geographic Information Engineering from Wuhan University, China. His research interests are in the areas of web cartography, outdoor augmented reality, spatial information sharing engineering and carto-linguistics.

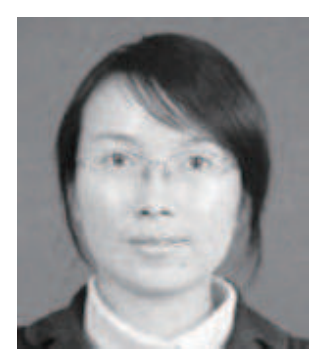

Xueling Wu is Lecturer of Institute of Geophysics and Geomatics at China University of Geosciences, Wuhan, China. She received the MA and $\mathrm{PhD}$ degree in Cartography and Geographic Information Engineering at Wuhan University, China. She finished her post-doctoral study in Surveying, Mapping Science and Technology at China university of Geosciences, Wuhan, China. Her research interests are in the areas of geo-information science and technology including location based service, outdoor augmented reality, landslide prediction and forecast. 